\title{
Smartphone und Depression: Ursache oder Therapie?
}

\author{
M. Spitzer, Ulm
}

Junge Menschen lieben ihr Smartphone und nutzen es täglich einige hundert Mal. Sie haben Angst, etwas zu verpassen (man spricht von Fear of Missing Out, kurz FoMO) oder gar, ihr Mobiltelefon oder zumindest dessen Anschluss zu verlieren (No more phone phobia, kurz Nomophobie), werden abgelenkt und leiden bei längerfristiger häufiger Nutzung mit erhöhter Wahrscheinlichkeit an Phantomempfindungen (50), Aufmerksamkeitsstörung und/oder Depression. Die bloße Anwesenheit des Smartphones auf dem Schreibtisch vermindert den IQ und das Denkvermögen (wozu erst kürzlich eine Übersicht in dieser Zeitschrift publiziert wurde; 49).

Dies überrascht zunächst. Schließlich heißt erstens das Englische Wort smart auf Deutsch schlau, und zweitens gehören soziale Online-Medien zu den meistgenutzten Smartphone-Anwenderprogrammen man spricht von Apps - überhaupt. Und weil Menschen sehr soziale Wesen sind, d. h. Glück und Lebenszufriedenheit oft über Sozialkontakte vermittelt erlebt werden, sollte die Smartphone-Nutzung mit einer höheren Lebensqualität und vor allem mit weniger negativen Emotionen einhergehen. Smartphones machen also weder schlau noch glücklich, sondern bewirken das genaue Gegenteil: Sie vermindern den Schulerfolg und - über soziale Online-Medien - vermindern das Wohlbefinden, die Zufriedenheit und führen zu mehr negativen Affekten wie Angst und Depression $(39,60)$. Man spricht sogar schon von Facebook-Depression oder von Smartphone-Depression.

Dass gerade die Nutzung des Smartphones hier Linderung verschaffen soll, wie zu-

Nervenheilkunde 2018; 37: 7-15

Korrespondenzadresse

Prof. Dr. Dr. Manfred Spitzer, Universitätsklinikum Ulm Klinik für Psychiatrie und Psychotherapie III

Leimgrubenweg 12, 89075 Ulm weilen behauptet wird (28), oder zumindest nichts mit der Entstehung einer Depression zu tun hat, wie ebenfalls zuweilen behauptet wird (15), erscheint angesichts der genannten Befunde mindestens fragwürdig.

\section{In Deutschland besitzen mehr als 95\% der Jugendlichen ein Smartphone. Seine Nutzung in entwickelten Ländern durch Jugendliche liegt bei gut drei bis mehr als fünf Stunden täglich.}

Beworben wird das Smartphone jedenfalls mit seinen vielen tollen Eigenschaften, die einem jungen Menschen von heute das Leben so sehr erleichtern sollen, dass er oder sie sich ein Leben ohne Smartphone gar nicht mehr vorstellen kann. Entsprechend besitzt hierzulande mittlerweile auch nahezu jeder (mehr als 95\%; 57) Jugendliche ein solches Gerät. Es wird - je nach Zeit der Studie und Land - von jungen Leuten zwischen dreieinviertel (Großbritannien, 2017) und fünfeinhalb Stunden (Südkorea, 2016) täglich benutzt. In den USA beträgt der Wert etwa vier Stunden (16), in Deutschland dürfte der Wert ebenfalls bei über drei Stunden liegen ${ }^{1}$. Ein Leben ohne Smartphone? - Für viele unvorstellbar! $\mathrm{Zu}$ weilen aber wird das Leben durch das Smartphone auch zur Hölle, wie Berichte über Mobbing, ungezügelte (anonyme) Aggressivität, gebrochenes Vertrauen, Datenklau, Facebook-Scheidung oder eben

1 Konkrete, verlässliche und vor allem neue Zahlen für 2017 hierzu sind kaum publiziert. Die diesbezüglichen Studien überschlagen sich vielmehr in Beschwichtigungen („Kinder lesen auch Bücher gerne") und verbergen wichtige Zahlen oft in einem Wust von Details und Interpretationen. Die Fakten sind jedoch recht eindeutig: 1. Schon für 2015 wurde die Online-Zeit von Kindern mit ca. vier Stunden angegeben. 2. Die meisten Online-Aktivitäten werden über das Smartphone abgewickelt. 3. Beide Tendenzen sind seit Jahren ansteigend.
Smartphone-Depression zeigen. - Wie ist es denn nun wirklich?

Diese Frage mag manchem zum Intellektualisieren neigenden, kritischen Zeitgenossen eigenartig erscheinen: „Wie kann man so naiv sein und so etwas fragen? Schließlich setzt diese Frage voraus, dass es eine Wirklichkeit gibt, aber das ist doch alles nur kulturell überformtes Konstrukt! Man kann doch nicht so tun, als könne man Sätze mit Sachen vergleichen, wie es diese naive Korrespondenztheorie der Wahrheit voraussetzt." Wer so denkt, wird weiter argumentieren, dass man lediglich Sätze miteinander vergleichen könne, also z. B. den Satz „es regnet hier jetzt" mit dem Satz "es regnet hier jetzt nicht" - nur einer kann wahr sein. Diese Kohärenztheorie der Wahrheit scheitert jedoch spätestens seit klar ist,

- dass man manches gar nicht beweisen kann oder

- dass wir (auch in der Wissenschaft) mit Widersprüchen leben (müssen).

Es bleibe also dem redlichen Intellektuellen nichts als eine pragmatische Wahrheitstheorie übrig, nach der sich - vorsichtig formuliert - manche manchmal darauf einigen können, was man (vielleicht) wahr nennen darf.

Ist Ihnen schon einmal aufgefallen, dass die Antwort auf Fragen wie „Herr Doktor, ist es Krebs?"“, „Herr Kapitän, gehen wir unter?" oder „Herr Sparkassendirektor, bin ich pleite?" nie darin besteht, die vermeintlich einzige intellektuell redliche Gegenfrage „welche Wahrheitstheorie setzen Sie voraus?" zu stellen? - Die Beispiele zeigen vielmehr aus meiner Sicht recht klar: Bei der Wahrheit geht es darum, was Sache ist - was jeden ehrlich und ernsthaft Fragenden zum "Korrespondenztheoretiker" macht! Und sollte er Zweifel an der Antwort des Spezialisten haben, dann fragt er weiter nach der Evidenz für dessen Aussagen - und die kann ein simples Faktum 


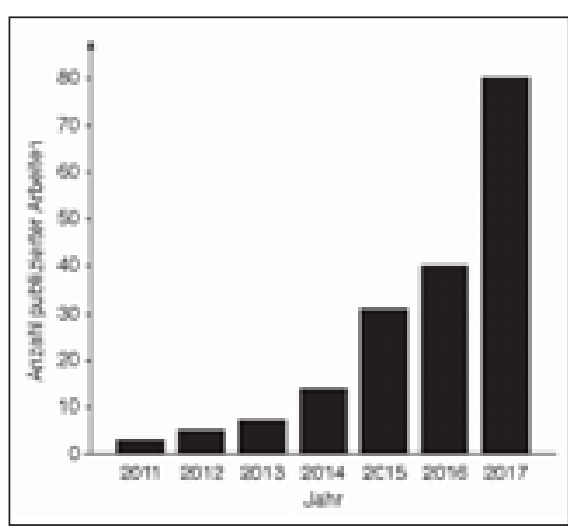

Abb. 1 Häufigkeit der Publikationen zu den Suchbegriffen "Smartphone" und "Depression" bei der medizinischen Datenbank pubmed in den Jahren 2011 (erste drei Einträge) bis 2017 (80 Einträge).

sein („mir jedenfalls steht das Wasser schon bis zum Hals"), eine Theorie („diese Schatten auf ihrem Röntgenbild sprechen für einen bösartigen Tumor") oder eine Mischung aus beidem („nach dem letzten Eintrag in Ihrem Sparbuch und der Theorie der Betriebswirtschaft: ja") sein.

Bei der Wahrheit geht es also um Korrespondenz und Kohärenz - wer einen Widerspruch behauptet, sagt gar nichts („,it's not even wrong", wie man im englischen manchmal hört) - und auch die Pragmatik ist im Spiel: Ginge es nicht um etwas Wichtiges - Krankheit, Untergang, Armut würde mancher tatsächlich ewig diskutieren.

Spätestens seit der Verbannung der Phrasen „evidenzbasiert" und „wissenschaftlich begründet" aus Forschungsanträgen für 2018 durch das Gesundheitsministerium der Trump-Administration am 15. Dezember $2017(7,25,51)$ sollte dem letzten allzu bescheidenen Intellektuellen klar sein, dass Zaghaftigkeit im Hinblick auf die Wahrheit dem durch die Vernunft geleiteten Handeln wenig dienlich ist! Vielmehr sollte jeder stolz darauf sein, dass unsere gesamte Kultur evidenzbasiert funktioniert, denn nur deswegen besteigen wir Flugzeuge, lassen uns narkotisieren und operieren, oder verlassen uns auf die Schmerztablette genau so wie auf das Navi im Auto - ohne darüber jeweils weiter nachzudenken!
Wie steht es also wirklich um den

Zusammenhang zwischen

Smartphone und Depression?

Wie steht es also wirklich um den Zusammenhang zwischen Smartphone und Depression? Schon ein flüchtiger Blick in die medizinische Fachliteratur scheint nahezulegen, dass seit einigen Jahren der Zusammenhang zwischen der Nutzung des Smartphones einerseits und einer erhöhen Wahrscheinlichkeit, an einer Depression zu erkranken andererseits, in zunehmendem Maße diskutiert wird ( Abb. 1).

Aber so einfach ist die Sache nicht, denn nicht wenige Einträge - vor allem solche aus den vergangenen zwei Jahren - beschäftigen sich nicht mit der SmartphoneDepression, sondern mit Versuchen, das Smartphone zur Diagnose und Therapie der Depression einzusetzen. Was ist davon zu halten? Ist das Smartphone nun Ursache der Depression oder deren Therapie? Wird hier vielleicht der Teufel mit dem Beelzebub ausgetrieben? ${ }^{2}$

Die Frage ist keineswegs nur von akademischem Interesse. Denn zum einen ist die Depression eine der häufigsten Krankheiten überhaupt, und jede Erhöhung ihrer Auftretenswahrscheinlichkeit ist entsprechend ernst zu nehmen, sind doch sehr viele Menschen davon betroffen. Betrachten wir die Zahlen zu Deutschland: Jedes Jahr erkranken in Deutschland gut fünf Millionen Menschen an einer (unipolaren) Depression. Wenn nun die Nutzung von Smartphones das Risiko, an einer Depression zu erkranken, um 10\% erhöhen würde, dann wären das allein in Deutschland eine halbe Million Kranke mehr! Dies ist kein Gedankenexperiment, wie die folgenden Fakten zeigen:

- Mädchen, die im Alter von 13 Jahren mehr als drei Stunden täglich in Face-

\footnotetext{
2 Vgl. die Evangelien von Markus 3,22, Matthäus 12, 24-27 und Lukas 11,15: Die Pharisäer werfen Jesus vor, er treibe den Teufel mit dem Beelzebub aus. Gemeint ist, dass ein Übel mit einem vielleicht noch größeren Übel getauscht wird. Beelzebub ist nicht nur ein jüdischer Name für „Teufel“, sondern auch für „bösen Dämon“, „Herr der Fliegen“ bzw. „Herr des Misthaufens“. Die Etymologie des Wortes ist sehr interessant, weil sich hier Bedeutungen und Laute verschieben und zudem mit Verballhornungen mischen.
}

book sind, haben mit 18 die doppelte Chance, an einer Depression erkrankt $\mathrm{zu}$ sein, wie eine britische Studie an knapp 1000 Jugendlichen gezeigt hat (37). Da $11 \%$ aller Mädchen in Großbritannien drei Stunden oder mehr täglich in Facebook sind, muss man diese Erkenntnis ernst nehmen.

- Die Häufigkeit von Suiziden hat sich in den USA zwischen den Jahren 2007 und 2015 bei jugendlichen Mädchen verdoppelt (von 2,4 auf 5,1 pro 100000), wie eine Untersuchung des Center for Disease Control in den USA gezeigt hat (4). Nach einer Analyse von Twenge et al. (56) lässt sich dieser Trend mit hoher Wahrscheinlichkeit mit der stark vermehrten Nutzung von Smartphones und Social Media in Zusammenhang bringen.

Zum Zweiten hat das soziale Online-Netzwerk Facebook kürzlich außerhalb Europas einen "Service" eingeführt, der darin besteht, dass mit Algorithmen in den Einträgen der Nutzer automatisch nach Hinweisen für Suizidalität gesucht wird, und diese Hinweise dann von den Computern bei Facebook mittels "machine learning" mit anderen Daten des Nutzers verbunden werden. So entstehen im Laufe der Zeit in den Computern von Facebook "Muster“ von Verhaltensweisen, die mit Suizidalität in Zusammenhang stehen und nach denen gesucht werden kann. Wenn dann im Einzelfall der Computer fündig wird, erscheint auf dem Bildschirm des Nutzers: „Jemand denkt, du brauchst Hilfe." Facebook möchte und kann mittlerweile $e^{3}$ damit frühzeitig Selbstmordabsichten identifizieren und im Ernstfall Hilfe durch Freunde oder Psychologen organisieren. Der Beweggrund hierfür waren in der Presse weithin publizierte Fälle von erfolgten Suiziden, die live in Facebook per Video zu sehen waren: Vor etwa einem Jahr filmte eine Zwölfjährige in den USA ihren eigenen Suizid und postete

\footnotetext{
3 Im März 2017 wurde das System der Öffentlichkeit präsentiert. Möglicherweise wurde es tatsächlich auch erst seit diesem Zeitpunkt aktiviert, wahrscheinlicher ist jedoch, dass es schon länger lernt, würde doch Facebook eher nicht einen "Service“ erst ankündigen und dann nachsehen, ob er auch funktioniert.
} 
das Video live. Etwa einen Monat später tat dies eine Vierzehnjährige, und im Oktober 2017 erschoss sich in der Türkei ein 54-jähriger Familienvater vor laufender Kamera, weil sich die Tochter ohne seine Erlaubnis verlobt hatte $(13,41)$.

\section{Erfolgte Suizide waren live in Facebook per Video zu sehen.}

Facebook hat bis in den Herbst 2017 hinein seine Algorithmen ausgiebig getestet und nach eigenen Angaben über 100 suizidale Nutzer durch spezielles Personal kontaktiert oder sogar aufsuchen lassen. Die Funktion der Erkennung von Suizidalität lässt sich nicht abschalten, man entkommt ihr also nicht. „Facebook geht es dabei natürlich nicht nur um den Schutz seiner Nutzer, sondern auch um die eigene ,weiße Weste", bemerkte hierzu die ARD-Tagesschau am 28. November 2017 (41). Denn es schade dem Image, wenn ein Unternehmen immer wieder mit ausgeführten Selbsttötungen in Verbindung gebracht wird.

Man kann diese Entwicklung positiv bewerten, wie beispielsweise Jakob Henschel vom Vorstand der Deutschen Gesellschaft für Suizidprävention: „Wenn Facebook die Posts der Nutzer ohnehin für Werbe- und Marktforschungszwecke analysiert, ist es nicht verkehrt, die Daten dann wenigstens auch für Hilfe in Krisen zu nutzen“" (zit. nach 13). Man kann den „Service“ aber auch für ein Trojanisches Pferd halten, mit dem der „Dienstleister“ Facebook noch tiefer in unserem Privatleben herumstochern will. Dieser Verdacht wird auch durch die Tatsache bestärkt, dass Facebook-Gründer Mark Zuckerberg den „Service“ in Europa wegen des hier gültigen viel strengeren Schutz der Privatsphäre nicht eingeführt hat, zugleich aber durchblicken ließ, dass er dies gerne tun würde, wenn sich Europa dafür entscheiden könnte, seine hohen Standards für den Schutz der Privatsphäre zu lockern. Machen wir uns also nichts vor: Für Herrn Zuckerberg geht es ums Geschäft!

4 Die Beispiele sind dem 2016 im Fachblatt Nature erschienenen News Feature Pocket Psychiatry entnommen (2, S. 20).
Nicht anders ist das mit den vielen Apps, die sich mittlerweile um das Thema Depression ranken: Zum „testen“, ob man depressiv ist („Depression Test“), zum „positiven Denken“" ("Think more positive!", „Optimism") oder zur Therapie („the easiest and most effective way to rewire your brain in just five minutes a day"). ${ }^{4}$ Sie sind zwar fast alle „kostenlos", aber wir haben ja verstanden, dass dies nur bedeutet, dass wir in einer anderen Währung als unserem Geld bezahlen: mit Informationen über uns selbst. ${ }^{5}$ Aber handelt es sich bei diesen auf dem Daumenkinobildschirm unseres Taschentelefons lauffähigen Programmen wirklich um den digitalen Rettungsanker („digital lifeline“) oder den Psychotherapeuten für die Westen- oder Hosentasche („portable therapist in every pocket") für jedermann („we can now reach people that up until recently were completely unreachable")? Sogar die Weltgesundheitsorganisation (WHO) schien das jedenfalls vor ein paar Jahren noch zu glauben, beinhaltet doch ihr Aktionsplan für die Jahre 2013 bis 2020 die Empfehlung, „die Selbstbehandlung beispielsweise durch die Nutzung von elektronischen und mobilen Gesundheitstechnologien zu unterstützen“?

Die vielen Apps zur Depression sind zwar fast alle "kostenlos", aber wir haben ja verstanden, dass dies nur bedeutet, dass wir in einer anderen Währung - nämlich mit Informationen über uns selbst - bezahlen.

Bevor hier jedoch öffentliche Gelder fließen und womöglich in den Sand gesetzt werden, wäre es hilfreich, zunächst nach der Evidenz für deren Nutzung zu fragen. Mittlerweile gibt es über 120 Apps zur Suizidprävention, die jedoch bislang kaum wissenschaftlich untersucht wurden, wie

5 „If you are not paying anything, you are not the customer but the product sold." Es ist zwar unklar, wer dies wann zuerst gesagt hat, aber der Ausspruch macht den Sachverhalt auf jeden Fall kurz und knapp sehr deutlich.

6 Auch diese Zitate entstammen oben bereits erwähnten Nature News Feature (2, S. 21).

7 WHO Zitat im Originaltext: "the promotion of selfcare, for instance, through the use of electronic and mobile health technologies" (nach 2, S. 21). drei Übersichtsarbeiten zu Smartphonebasierten Suizidpräventions-Programmen zeigen $(9,32,59)$. Die Wirkungen und Nebenwirkungen dieser vermeintlichen mobilen Helferlein in der Hosen- oder Westentasche sind also praktisch unbekannt, weswegen deren Nutzung von den Autoren der drei Arbeiten zu SuizidpräventionsApps nicht empfohlen wird. Auch eine kürzlich publizierte Übersicht zu Smartphone Technologies and [...] depression (18) kommt zum gleichen Ergebnis.

In dem erwähnten Nature-Kommentar - betitelt Pocket Psychiatry. Mobile mentalhealth Apps have exploded onto the marked [...] - kann man nachlesen, dass sich die Digitalwirtschaft wieder einmal deutlich schneller bewegt als die Wissenschaft (2). Der Chairman der Smartphone App Evaluation Task Force der American Psychiatric Association jedenfalls wird mit den Worten zitiert, dass sich das Ganze im Moment wie der Wilde Westen der Gesundheitsversorgung anfühle (2, S. 21). ${ }^{8}$

Solche Zustände wie im wilden Westen kennt man aus anderen Bereichen wie beispielsweise der Pädagogik. Da wird das „digitale Lernen“" von Marktschreiern ohne jeden wissenschaftlichen Hintergrund - d. h. ohne dass es Daten gäbe, die das Vorgehen empirisch begründen und damit rechtfertigen könnten - propagiert, obwohl dadurch die Bildung und die Gesundheit von Kindern ruiniert wird. Wer das nicht glaubt, besuche die Didacta, nach eigenen Angaben „die weltweit größte Fachmesse rund um Bildung".9 Dort geht es längst nicht mehr darum, wie Kinder lernen, sondern darum, wie man verängstigten Eltern und verunsicherten Lehrern mit ein bisschen banalem „Edutainment" den Elektroschrott von Morgen verkauft.

8 Auch hierzulande gibt es die Task Force E-Health der DGPPN, die einen Kriterienkatalog vorgeschlagen hat, anhand dessen „überprüft werden kann, ob eine internetbasierte Intervention sich für die Behandlung psychischer Störungen im Rahmen der klinischen Versorgung in Deutschland eignet" (32, S. 1191).

9 www.messe-stuttgart.de/didacta/ 
Wenn der aus der Pädagogik bekannte digitale Hype auf die Medizin überschwappen sollte, wäre das mehr als peinlich.

Wenn nun derartiger aus der Pädagogik bekannter digitaler Hype auf die Medizin überschwappen sollte, wäre das mehr als peinlich. Das Ausmaß der Beeinträchtigung der Gesundheit der weltweit mittlerweile 3,8 Milliarden Smartphone-Nutzer rechtfertigt längst die Rede von einer Smartphone-Epidemie, macht doch dieses Gerät uns nachweislich in vielfacher Hinsicht krank. Smartphones verursachen Übergewicht (29), Haltungsschäden (44), Unfälle, Schlafstörungen (47) Kurzsichtigkeit (48) und Sucht (45) -, sowohl nach dem Smartphone als auch nach anderen bekannten Suchtstoffen wie Alkohol und Nikotin ( $\$ Tab. 1). Hinzu kommen Wechselwirkungen zwischen diesen Effekten, wie beispielsweise eine prädiabetische Stoffwechsellage durch Schlafmangel, die die Auswirkungen von Übergewicht verstärkt. Typ-II-Diabetes wiederum ist bei Depression erhöht - und umgekehrt (38). Ein drittes Beispiel: Internet-Konsum wurde mit erhöhtem Blutdruck in Verbindung gebracht, was das erhöhte kardiovaskuläre Risiko von Adipositas verstärkt.

$\mathrm{Zu}$ alledem kommen die eingangs bereits erwähnten Störungen im geistig-seelischen Bereich sowie Lernschwierigkeiten und Schulprobleme (46), dadurch bedingte geringere Bildung und dadurch wiederum bedingte erhöhte Wahrscheinlichkeit von Demenz im Alter. ${ }^{10}$

Dass diese Technik heutzutage als modernster Gesundheitsbringer verkauft wird, ist etwa so, als würde die Medizin jetzt weltweit vom Zucker als neues Wunderheilmittel sprechen, weil Zucker bei Diabetikern im Unterzucker tatsächlich Gutes bewirken kann. „Die Dosis macht das Gift“, muss man hier Paracelsus wieder einmal zitieren, und zugleich realisieren, dass wir beim

10 Bei allen molekularen Erkenntnisfortschritten wird gerne die grundlegende Einsicht vergessen, dass De-Mens (lateinisch: herab - Geist) ganz allgemein geistigen Abstieg meint. Für jeden Abstieg gilt: Je höher man beginnt, desto länger dauert es, bis man unten ist. Entsprechend ist die (in jungen Jahren erreichte) Bildung eines Menschen der stärkste protektive Faktor gegenüber einer Demenz im Alter (35).

Tab. 1 Risiken und Nebenwirkungen von Smartphones.

\begin{tabular}{|c|c|c|}
\hline Körperlich & Geistig-seelisch & Gesellschaftlich \\
\hline Bewegungsmangel & Angst (FoMO, Nomophobie) & geringere Bildung \\
\hline Adipositas & Mobbing & geringeres gegenseitiges Vertrauen \\
\hline Haltungsschäden & $\begin{array}{l}\text { Aufmerksamkeitsstörungen } \\
\text { (ADHD) }\end{array}$ & $\begin{array}{l}\text { verminderte Fähigkeit zur } \\
\text { Willensbildung }\end{array}$ \\
\hline $\begin{array}{l}\text { Diabetes (durch } \\
\text { Bewegungsmangel, } \\
\text { Adipositas und Insomnie) }\end{array}$ & Demenz & $\begin{array}{l}\text { weniger Naturerleben und damit } \\
\text { geringere Förderung von } \\
\text { Nachhaltigkeit }\end{array}$ \\
\hline Hypertonie & $\begin{array}{l}\text { Depression, einschließlich } \\
\text { Suizidalität }\end{array}$ & mehr Anonymität \\
\hline Myopie & Empathieverlust & weniger Solidarität \\
\hline Insomnie & $\begin{array}{l}\text { verminderte } \\
\text { Lebenszufriedenheit }\end{array}$ & $\begin{array}{l}\text { mehr soziale Isolation und } \\
\text { Einsamkeit }\end{array}$ \\
\hline Risikoverhalten: Unfälle & $\begin{array}{l}\text { Sucht: Alkohol- und } \\
\text { Drogensucht }\end{array}$ & $\begin{array}{l}\text { geringere Gesundheit der } \\
\text { Bevölkerung }\end{array}$ \\
\hline $\begin{array}{l}\text { Risikoverhalten: } \\
\text { Geschlechtskrankheiten }\end{array}$ & $\begin{array}{l}\text { Sucht: Smartphone- und } \\
\text { Online-Spiele-Sucht }\end{array}$ & Gefährdung der Demokratie \\
\hline
\end{tabular}

Smartphone gerade was unsere nächste Generation anbelangt eine hoffnungslose Überdosierung zulassen - mit all ihren Risiken und Nebenwirkungen ( $\$$ Tab. 1). Und warum tun wir das? Weil die reichsten Firmen der Welt eine gewaltige Lobby-Arbeit betreiben, um noch reicher zu werden. Dass dabei die körperliche und seelische Gesundheit sowie die Bildung der nächsten Generation leidet, ist diesen Firmen egal: Es geht schließlich um Milliarden. Dies trifft für die Pharmaindustrie auch $\mathrm{zu}$, aber im Pharma-Bereich sind die Dinge sehr klar geregelt: Die Wirkungen müssen nachgewiesen und die Nebenwirkungen bekannt und als vergleichsweise gering bewertet werden, damit eine neue medizinische Prozedur auf den Markt kommen darf.

\section{"Die Dosis macht das Gift" - mit Paracelsus muss man einsehen, dass wir beim Smartphone eine hoffnungslose Überdosierung zulassen.}

Betrachtet man die Wirkungen und Nebenwirkungen des Smartphones unter diesem Gesichtspunkt, dann ergibt sich sehr klar: Im Vergleich zur Evidenz für die deutlich gesundheitsrelevanten Risiken und Nebenwirkungen nimmt sich die Evidenz für die Wirkungen bescheiden aus: Mehr als kleine, bislang nicht replizierte Studien, die meist Pilotcharakter hat- ten und zudem auch noch von den Herstellern der Apps finanziert und durchgeführt wurden, gibt es nicht. Das sagen auch die mittlerweile vorliegenden Reviews und Metaanalysen zum Thema Smartphone und Gesundheit im Allgemeinen sowie zu Smartphone-Apps bei Depression im Besonderen (12).

Dem anfänglichen Enthusiasmus - auch bei der Weltgesundheitsorganisation (WHO) und dem britischen National Health Service (NHS) - folgte die Ernüchterung: Nachdem man eine Liste von „sicheren und vertrauenswürdigen" Apps publiziert hatte, ergab eine Untersuchung der 14 zur Behandlung von Depression und Angst angeführten Apps, dass nur für vier davon irgendeine Form von Evidenz zur Effektivität vorlag. Die Autoren der Studie folgerten entsprechend: „[...] um zu gewährleisten, dass die Apps nicht mehr Schaden als Nutzen anrichten, ist es wichtig, dass die derzeit vom Nationalen Gesundheits-Service (NHS) empfohlenen Apps, die entweder keine beweiskräftigen Methoden zu ihrer Prüfung nutzten oder deren Prüfung keinen Anhalt für deren Effektivität ergab, von der Liste gestrichen werden ${ }^{\text {"11 }}$ (34). Das

$11,[\ldots]$ in order to ensure that apps don't do more harm than good, it is important that those presently recommended by the NHS apps library, that either fail to demonstrate evidence underlying the methodological approach taken, or evidence of effectiveness in use, are removed." 
Tab. 2 Studien zur Smartphone-Nutzung und Depression (S: Smartphone)

\begin{tabular}{|c|c|c|c|}
\hline Autor, Jahr & Land & $\begin{array}{l}\mathrm{N} \text {, untersuchte Per- } \\
\text { sonen }\end{array}$ & Ergebnis \\
\hline $\begin{array}{l}\text { Aker et al. } 2017 \\
\text { (1) }\end{array}$ & Türkei & 495 Studenten & $\begin{array}{l}\text { S-Sucht geht mit Angst, Schlafstörungen } \\
\text { und Depression einher }\end{array}$ \\
\hline $\begin{array}{l}\text { Chen et al. } 2017 \\
\text { (5) }\end{array}$ & China & $\begin{array}{l}1441 \text { Studenten } \\
\text { ( } 51,7 \% \text { weibl. mittle- } \\
\text { res Alter } 19,7 \text { Jahre) }\end{array}$ & $\begin{array}{l}\text { S-Sucht bei ca. 30\% der Studenten; bei } \\
\text { Männern mit Spiele App-Nutzung, Angst und } \\
\text { Schlafstörungen assoziiert, bei Frauen hinge- } \\
\text { gen mit der Nutzung sozialer Online-Netzwer- } \\
\text { ke, Angst, Schlafstörungen und Depression }\end{array}$ \\
\hline $\begin{array}{l}\text { Choi et al. } 2015 \\
\text { (6) }\end{array}$ & Südkorea & 448 Studenten & $\begin{array}{l}\text { S-Sucht korreliert negativ mit depressiven } \\
\text { Symptomen bei Gesunden }\end{array}$ \\
\hline $\begin{array}{l}\text { Demirci et al. } \\
2015 \text { (11) }\end{array}$ & Türkei & $\begin{array}{l}319 \text { Studenten ( } 203 \\
\text { weibl, mittleres Alter } \\
\text { 20,5 Jahre) }\end{array}$ & $\begin{array}{l}\text { high S-User }(n=127) \text { im Vergleich zu low } \\
\text { S-Usern }(n=121) \text { : mehr Depressivität, } \\
\text { Angst, Schlafstörungen und Smartphone- } \\
\text { Sucht }\end{array}$ \\
\hline $\begin{array}{l}\text { Gao et al. } 2017 \\
\text { (19) }\end{array}$ & China & $\begin{array}{l}722 \text { Studenten } \\
\text { ( } 71,1 \% \text { weibl. mittle- } \\
\text { res Alter } 20,5 \text { Jahre) }\end{array}$ & $\begin{array}{l}\text { deutlicher Zusammenhang zwischen } \\
\text { Smartphone-Nutzung und sowohl } \\
\text { Depression als auch Neurotizismus }\end{array}$ \\
\hline $\begin{array}{l}\text { Kim E et al. } 2017 \\
(28)\end{array}$ & Südkorea & 200 Studenten & $\begin{array}{l}\text { signifikanter Zusammenhang zwischen } \\
\text { S-Sucht und Depression }\end{array}$ \\
\hline $\begin{array}{l}\text { Kim HJ et al. } \\
2017 \text { (27) }\end{array}$ & Südkorea & 608 Studenten & $\begin{array}{l}\text { deutlicher Zusammenhang zwischen } \\
\text { S-Nutzung und Depression }\end{array}$ \\
\hline $\begin{array}{l}\text { Matar Boumos- } \\
\text { leh, Jaalouk } \\
2017 \text { (37) }\end{array}$ & Libanon & $\begin{array}{l}688 \text { Studenten }(47 \% \\
\text { weibl, mittleres Alter } \\
20,6 \text { Jahre) }\end{array}$ & $\begin{array}{l}\text { höhere S-Nutzung ging mit mehr Angst, } \\
\text { Depressivität, Schlafstörungen und Smartpho- } \\
\text { ne-Sucht einher; zudem mit Typ-A-Persönlich- } \\
\text { keit, jüngerem Lebensalter bei Beginn der } \\
\text { S-Nutzung, mehr S-Gebrauch zur Unterhaltung } \\
\text { und weniger Kontakten zur Familie }\end{array}$ \\
\hline $\begin{array}{l}\text { Sohn et al. } 2017 \\
\text { (44) }\end{array}$ & Südkorea & 416 Schüler & $\begin{array}{l}\text { Mobbing und Depressivität steht in } \\
\text { deutlichem Zusammenhang mit erhöhter } \\
\text { Suizidalität, Smartphone-Sucht auch } \\
\text { (2,4-fach erhöhte Suizidalität) }\end{array}$ \\
\hline $\begin{array}{l}\text { Thomée et al. } \\
2012 \text { (53) }\end{array}$ & Schweden & $\begin{array}{l}1127 \text { (19-25 Jahre) } \\
4163 \text { (20-25 Jahre) }\end{array}$ & $\begin{array}{l}\text { dauernde Erreichbarkeit durch Handy und } \\
\text { Nutzung am Abend führt besonders zu } \\
\text { Schlafstörungen und Depression, vor allem } \\
\text { bei Frauen }\end{array}$ \\
\hline $\begin{array}{l}\text { Wolniewicz et al. } \\
2017 \text { (61) }\end{array}$ & USA & $\begin{array}{l}295 \text { Studenten } \\
\text { ( } 57,1 \% \text { weibl. mittle- } \\
\text { res Alter } 20 \text { Jahre) }\end{array}$ & $\begin{array}{l}\text { hochsignifikante Korrelation zwischen } \\
\text { S-Nutzung und negativem Affekt }\end{array}$ \\
\hline
\end{tabular}

geschah dann auch, nicht nur beim britischen NHS, sondern auch bei der (globalen) WHO. Denn gerade die Empfehlungen bekannter öffentlicher Behörde werden von vielen Menschen kaum hinterfragt und ernst genommen. Zudem führen unbewiesene Behauptungen oft zu unbegründeten Hoffnungen gerade bei denjenigen, die besonders verletzlich sind und wenig für ihr Schicksal können: ängstliche, sozial schwache bzw. benachteiligte Menschen. Es ist also ganz ähnlich wie in der Pädagogik, wo es immer Kin- das höchste Maß an Bedürftigkeit und die geringsten Zugangsmöglichkeiten zu wirksamen Therapien aufweisen", 12 schreiben die Autoren der Studie (33, S. 98), die letztlich dazu geführt hat, dass Empfehlungen zurückgezogen wurden. Im Hinblick auf das Erziehungswesen provoziert dies durchaus die Frage: Wann und wo geschah dies - das Eingeständnis falscher Entscheidungen seitens der Behörden angesichts neuer wissenschaftlich begründeter Tatsachen - jemals in der Pädagogik? ${ }^{13}$ - die Antwort auf diese Frage macht den Mediziner mit Recht stolz und sollte allen Pädagogen Grund zum Nachdenken sein!

\section{Die Mehrheit der Gesundheits-Apps gibt private Daten unverschlüsselt auf die Server der Anbieter weiter.}

Neben der Depression (zu der es über 1500 Smartphone-Apps gibt) findet sich eine ganze Reihe von Krankheitsbildern, zu deren Diagnose und/oder Therapie mittlerweile Apps vorliegen. Zum Diabetes beispielsweise wurden noch mehr Apps mehr als 1700 - entwickelt (35). Und obgleich dieses Krankheitsbild

- in seiner Pathophysiologie wesentlich besser verstanden ist als die Depression und

- klare, d. h. reliable und eindeutig interpretierbare Outcome-Variablen existieren (z. B. der Blutwert $\mathrm{HbA}_{1 c}$ ),

zeigen erste randomisierte, kontrollierte Studien zu Diabetes-Apps keinen Effekt $(20,58)$. Da wundert es kaum, dass die Dinge bei dem wesentlich komplexeren Sachverhalt der Depression nicht besser liegen: Smartphone-Apps zur Diagnose und Therapie der Depression versprechen viel und es gibt sie zu Hauf. Sie halten jedoch bislang nicht, was sie versprechen und beinhalten stattdessen Risiken und Nebenwirkungen. Hinzu kommen die be-

Dies darf ein funktionierendes Gemeinwesen nicht zulassen, und im Falle des Gesundheitswesens funktionieren die hierfür eingerichteten Mechanismen auch: „Der Kauf und die Nutzung von Apps, deren klinische Wirksamkeit noch nicht nachgewiesen wurde, stellt nicht nur eine mögliche Geldverschwendung dar, sondern kann auch zu einer Vergrößerung der Angst gerade bei denjenigen führen, die zugleich $12,[\ldots]$ the purchase and use of apps that are yet to demonstrate objective clinical benefit is not only a potential waste of money, but also likely to have a compounding effect on levels of anxiety in those with the greatest need and least access to effective treatment."

13 Warum ist uns unsere Gesundheit so sehr viel wichtiger als uns unsere Kinder sind? 
kannten Risiken und Nebenwirkungen des Smartphone-Gebrauchs selbst.

Im Bereich der Nervenheilkunde sind diese Erkenntnisse besonders relevant, liegt doch gerade bei psychisch kranken Menschen eine besonders hohe Vulnerabilität (Verletzlichkeit) vor im Hinblick auf Vertrauensbildung und Vertrauensverlust. Daher ist an dieser Stelle auch zu vermerken, dass Untersuchungen zur Datensicherheit bei Gesundheits-Apps ein erschreckendes Bild abgaben: Die Mehrheit der Produkte gab private Daten unverschlüsselt auf die Server der Anbieter weiter, oft ohne dies dem Nutzer mitzuteilen oder ihn zumindest auf das Ausmaß und die Tatsache der fehlenden Verschlüsselung hinzuweisen $(17,21,23,24)$. Eine Studie von 24405 (!) Mobile Health Apps ergab bei mehr als 95\% von ihnen mindestens eine Sicherheitslücke im Hinblick auf den Schutz der Privatheit der Daten (10).

Schließlich muss noch berücksichtigt werden, dass die Nutzung von Smartphones bekanntermaßen direkte negative Auswirkungen auf den Affekt hat ( Tab. 2). Eine kürzlich publizierte Studie aus den USA (60) an 295 Studenten (57,1\% weibl.) im Durchschnittsalter von 20 Jahren beispielsweise ergab nicht nur einen hoch signifikanten Zusammenhang der Smartphone-Nutzung mit Angst (FoMO), sondern auch mit negativem Affekt. Frauen waren insgesamt stärker betroffen als Männer. Dies liegt nicht zuletzt daran, dass Frauen sozialere Wesen sind, mehr soziale Medien nutzen, sich stärker mit anderen vergleichen und damit insgesamt noch anfälliger für die negativen Effekte von Medien wie Facebook sind.

Eine Studie aus dem Libanon (nach Kuweit dasjenige arabische Land mit den meisten Smartphones pro Kopf der Bevölkerung) ging dem Zusammenhang zwischen Smartphone-Gebrauch, Depression und Smartphone-Sucht bei 688 Studenten im Alter von 21 Jahren (53\% männlich) nach (36). Die Datenerhebung fand im Herbst 2014 und Frühjahr 2015 statt, wobei nicht nur das Ausmaß der SmartphoneNutzung, sondern auch Angst, Depression, Persönlichkeit, Smartphone-Sucht und eine Reihe anderer Variablen erfasst wurden. Das mittlere Alter beim Beginn der Smartphone-Nutzung lag bei 15,09 $( \pm 2,12)$

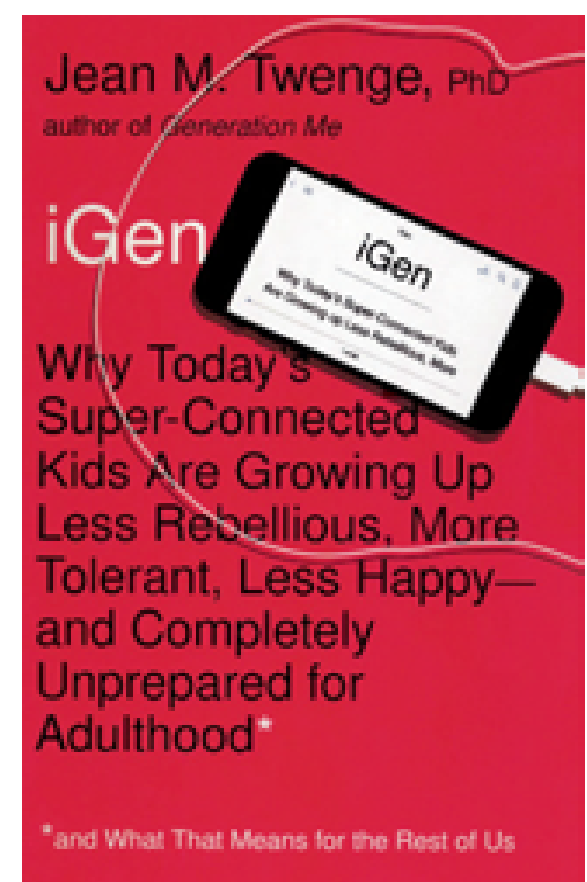

Abb. 2 Cover der sehr lesenswerten Monografie von Jean Twenge (55).

Jahren, eine exzessive Nutzung (fünf oder mehr Stunden täglich an Wochentagen) fand sich bei $49 \%$ der Befragten. Bei einem Viertel $(26,5 \% \%)$ der Studenten lagen Ängste vor, bei einem Fünftel $(21,8 \%)$ eine Depression. Eine stärkere SmartphoneNutzung ging mit mehr Angst, Depressivität, Schlafstörungen und SmartphoneSuch $t^{14}$ einher. Auch zeigte sich, dass eine Typ-A-Persönlichkeit (gekennzeichnet durch Aggressivität, Kompetitivität, Wut, Zynismus und geringes Vertrauen) im Gegensatz zu einer Typ-B-Persönlichkeit (charakterisiert durch Lockerheit, Ent-

14 Der Zusammenhang von Smartphone-Depression und Smartphone-Sucht ist nicht völlig geklärt: Einerseits findet man bei erhöhtem Smartphone-Gebrauch auch erhöhte Werte für Smartphone-Sucht und Smartphone-Depression, und bei der Untersuchung von Patienten mit Smartphone-Sucht findet man auch erhöhte Werte für eine Depression. Andererseits wurde in einer Studie aus Südkorea bei 448 Studenten (also nicht bei Patienten) ein negativer Zusammenhang zwischen dem Vorhandensein einer Sucht und dem Vorhandensein von depressiven Symptomen bei Gesunden beobachtet (6). Hierbei könnte es sich allerdings um ein Methodenartefakt (bias) handeln. Solche methodenbedingten Tendenzen (biases) sind für SmartphoneInterventionsstudien zur Verhaltenskontrolle häufig beschrieben (62). spanntheit und Geduld) eher mit höherem Smartphone-Gebrauch in Zusammenhang stand. Auch ein jüngeres Lebensalter bei Beginn der Smartphone-Nutzung, mehr Smartphone-Gebrauch zum Zeitvertreib und weniger Kontakte zur Familie (mit Hilfe des Smartphones) gingen mit einer erhöhten Smartphone-Nutzung einher. Ähnliche Ergebnisse hatten Studien aus Schweden (52), der Türkei (11), Südkorea $(26,27,43)$ und China (19) ergeben.

Die US-Psychologin Jean Twenge beschäftigt sich seit geraumer Zeit mit den Besonderheiten der jungen Menschen in ihrem Lande. Sie sieht einen klaren Zusammenhang zwischen der Nutzung von Smartphones und sozialen Medien wie Facebook und Twitter einerseits und der dramatischen Zunahme von Depressionen und Suiziden in den USA. Auch sie wird heftig dafür angegriffen, dass sie die Wahrheit sagt, mit den gleichen Argumenten, die mir seit der Publikation von Digitale Demenz immer wieder - und unbegründet - entgegengehalten werden (z. B. „sucht sich die Studien aus, verwechselt Korrelation mit Kausalität, sieht die Dinge zu negativ bzw. skeptisch“; 3, 22). Wer also mir nicht glaubt, der lese einfach ihr neues Buch (Abb. 2). Er oder sie kann - und sollte sowieso - alles selbst googeln, denn im Bereich der Wissenschaft geht es nicht darum, etwas zu glauben, sondern darum, etwas begründet zu wissen.

\section{Literatur}

1. Aker S, Şahin MK, Sezgin S, Oğuz G. Psychosocial factors affecting Smartphone Addiction in university students. J Addict Nurs 2017; 28: 215-219.

2. Anthes E. Pocket Psychiatry (News Feature). Nature 2016; 532: 20-23.

3. Cavanagh SR. No, Smartphones are not destroying a generation. Psychology Today, 6.8.2017 https://www.psychologytoday.com/blog/oncemore-feeling/201708/no-smartphones-are-not-destroying-generation

4. CDC, Center for Disease Control (2017) QuickStats: Suicide Rates for Teens Aged 15-19 Years, by Sex - United States, 1975-2015. MMWR Morb Mortal Wkly Rep 2017 66: 816.

5. Chen B, Liu F, Ding S, Ying X, Wang L, Wen Y. Gender differences in factors associated with smartphone addiction: a cross-sectional study among medical college students. BMC Psychiatry 2017; 17(1): 341.

6. Choi SW, Kim DJ, Choi JS, Ahn H, Choi EJ, Song WY, Kim S, Youn H. Comparison of risk and pro- 
tective factors associated with smartphone addiction and Internet addiction. J Behav Addict 2015; 4: 308-314.

7. Cohen J. CDC word ban? The fight over seven health-related words in the president's next budget. Posted in: Scientific Community (doi:10.1126/science.aar7959), $\quad 18.12 .2017$ http://www.sciencemag.org/news/2017/12/fightover-seven-health-related-words-presidents-next-budget

8. Collier R. Mental health in the smartphone era. CMAJ 2016; 188: 1141-1142.

9. de la Torre I, Castillo G, Arambarri J, López-Coronado M, Franco MA. Mobile apps for suicide prevention: Review of virtual stores and literature. JMIR Mhealth Uhealth 2017; 5(10): e130.

10. Dehling T, Gao F, Schneider S, Sunyaev A. Exploring the far side of mobile health: Information security and privacy of mobile health Apps on iOS and Android. JMIR Mhealth Uhealth 2015; 3(1): e8.

11. Demirci K, Akgönül M, Akpinar A. Relationship of smartphone use severity with sleep quality, depression, and anxiety in university students. J Behav Addict 2015; 4: 85-92.

12. Donker T, Petrie K, Proudfoot J, Clarke J, Birch MR, Christensen H. Smartphones for smarter delivery of mental health programs: A systematic review. J Med Internet Res 2013; 15(11): e247.

13. Eder S. Facebook und sein trojanisches Pferd. FAZ 20.3.2017 http://www.faz.net/aktuell/gesellschaft/ gesundheit/kuenstliche-intelligenz-untersuchtpsyche-von-facebook-nutzern14931226.html?printPagedArticle=true\#void

14. Elhai JD, Dvorak RD, Levine JC, Hall BJ. Problematic smartphone use: a conceptual overview and systematic review of relations with anxiety and depression psychopathology. J Affect Disord 2017; 207: 251-259.

15. Elhai JD, Levine JC, Dvorak RD, Hall BJ. Non-social features of smartphone use are most related to depression, anxiety and problematic smartphone use. Comput Hum Behav 2017; 69: 75-82.

16. Elhai JD, Tiamiyu MF, Weeks JW, Levine JC, Picard KJ, Hall BJ. Depression and emotion regulation predict objective smartphone use measured over one week. Personal. Individ Dif 2017 http://dx.doi.org/10.1016/j.paid.2017.04.051

17. Fernandez-Luque L, Staccini P. All that glitters is not gold: Consumer health informatics and education in the era of social media and health apps. Findings from the Yearbook 2016 Section on Consumer Health Informatics. Yearb Med Inform 2016; 1: 188-193.

18. Frank E, Pong J, Asher Y, Soares CN. Smart phone technologies and ecological momentary data: is this the way forward on depression management and research? Curr Opin Psychiatry 2018; 31: 3-6.

19. Gao T, Xiang YT, Zhang H, Zhang Z, Mei S. Neuroticism and quality of life: Multiple mediating effects of smartphone addiction and depression. Psychiatry Res 2017; 258: 457-461.

20. Goyal S, Nunn CA, Rotondi M, Couperthwaite AB, Reiser S, Simone A, Katzman DK, Cafazzo JA, Palmert MR. A mobile App for the self-management of Type 1 Diabetes among adolescents: A randomized controlled trial. JMIR Mhealth Uhealth 2017; 5: e82.
21. Grundy Q, Held FP, Bero LA. Tracing the potential flow of consumer data: A network analysis of prominent health and fitness apps. J Med Internet Res 2017; 19(6): e233.

22. Guernsey L. Don't Take Away Your Teen's Phone. Slate 10.8.2017 http://www.slate.com/articles/tech nology/future_tense/2017/08/smartphones_haven_t_destroyed_a_generation.html

23. He D, Naveed M, Gunter CA, Nahrstedt K. Security concerns in android mHealth apps. AMIA Annu Symp Proc 2014: 645-654.

24. Huckvale K, Prieto JT, Tilney M, Benghozi PJ, Car J. Unaddressed privacy risks in accredited health and wellness apps: a cross-sectional systematic assessment. BMC Med 2015; 13: 214.

25. Kaplan S, McNeil DG Jr. Uproar Over Purported Ban at C.D.C. of Words Like 'Fetus'. The New York Times 16.12.2017 https://www.nytimes. com/2017/12/16/health/cdc-trump-bannedwords.html

26. Kim E, Cho I, Kim EJ. Structural equation model of smartphone addiction based on adult attachment theory: Mediating effects of loneliness and depression. Asian Nurs Res (Korean Soc Nurs Sci) 2017; 11: 92-97.

27. Kim HJ, Min JY, Kim HJ, Min KB. Association be tween psychological and self-assessed health status and smartphone overuse among Korean college students. J Ment Health 2017; 4: 1-6.

28. Kim J, Seo M, David P. Alleviating depression only to become problematic mobile phone users: can face-to-face communication be the antidote? Comput Hum Behav 2015; 51: 440-447.

29. Kim SE, Kim JW, Jee YS. Relationship between smartphone addiction and physical activity in Chinese international students in Korea. J Behav Addict 2015; 4: 200-205.

30. Kim SK, Kim SY, Kang HB. An analysis of the effects of smartphone push notifications on task performance with regard to smartphone overuse using ERP. Comput Intell Neurosci 2016: 5718580.

31. Klein JP et al. Internetbasierte Interventionen in der Behandlung psychischer Störungen. Der Nervenarzt 2016; 87: 1185-1193.

32. Larsen ME, Nicholas J, Christensen H. A systematic assessment of smartphone tools for suicide prevention. PLoS ONE 2016; 11(4): e0152285.

33. Leigh S, Flatt S. App-based psychological interventions: friend or foe? Evidence-Based Mental Health 2015; 18: 97-99.

34. Livingston G, et al. Dementia prevention, intervention, and care. Lancet 2017; 390: 2673-2734.

35. Martínez-Pérez B, de la Torre-Díez I, López-Coronado $\mathrm{M}$. Mobile health applications for the most prevalent conditions by the World Health Organization: Review and analysis. J Med Internet Res 2013; 15(6): e120.

36. Matar Boumosleh J, Jaalouk D. Depression, anxiety, and smartphone addiction in university students - A cross sectional study. PLoS ONE 2017; 12(8): e0182239.

37. McTague T. Children glued to social media sites like Facebook and Twitter are twice as likely to suffer mental health problems. Daily Mail 20.10.2015 http://www.dailymail.co.uk/news/ar ticle-3281206/Children-glued-social-media-siteslike-Facebook-Twitter-TWICE-likely-suffer-mental-health-problems.html?ito=social-twitter_dailymailus
38. Mendenhall E, Kohrt BA, Norris SA, Ndetei D, Prabhakaran D. Non-communicable disease syndemics: poverty, depression, and diabetes among low-income populations. Lancet 2017; 389: 951-963.

39. Oberst U, Wegmann E, Stodt B, Brand M, Chamarro A. Negative consequences from heavy social networking in adolescents: The mediating role of fear of missing out. J Adolesc 2017; 55: 51-60.

40. Orth B. Die Drogenaffinität Jugendlicher in der Bundesrepublik Deutschland 2015. Teilband Computerspiele und Internet. BZgA-Forschungsbericht. Köln: Bundeszentrale für gesundheitliche Aufklärung 2017.

41. Schuler M. Facebook will Suizide verhindern. Tagesschau.de vom 28.11.2017 https://www.tagess chau.de/wirtschaft/facebook-suizidpraevention-101.html

42. Shen N, Levitan MJ, Johnson A, Bender JL, Hamilton-Page M, Jadad AA, Wiljer D. Finding a depression app: a review and content analysis of the depression app marketplace. JMIR Mhealth Uhealth 2015; 3(1): e16.

43. Sohn M, Oh H, Lee SK, Potenza MN. Suicidal ideation and related factors among Korean High School Students: A focus on cyber addiction and school bullying. J Sch Nurs 2017; 1: 1059840517734290.

44. Spitzer M. Smartphones. Zu Risiken und Nebenwirkungen für Bildung, Sozialverhalten und Gesundheit. Nervenheilkunde 2014; 33: 9-15.

45. Spitzer M. Cyberkrank! München: Drömer 2015.

46. Spitzer M. M-Learning? When it comes to learning, smartphones are a liability, not an asset. Trends in Neuroscience and Education 2015; 4 : 87-91.

47. Spitzer M. Schlaflos mit Blaulicht. Nervenheilkunde 2015; 34: 560-562.

48. Spitzer M. Kurzsichtig wegen mangelnder Weitsicht. Nervenheilkunde 2016; 35: 152-155.

49. Spitzer M. Die Smartphone-Denkstörung Nervenheilkunde 2017; 36: 587-590.

50. Spitzer M. Phantom-Vibration. Nervenheilkunde 2017; 36: 655-658.

51. Sun LH, Eilperin J. CDC gets list of forbidden words: Fetus, transgender, diversity. The Washington Post 15.12.2017 https://www.washingtonpost. $\mathrm{com} /$ national/health-science/cdc-gets-list-of-forbidden-words-fetus-transgender-diversity/2017/12/15/f503837a-elcf-

11e7-89e8-edec16379010_story.html?utm_term $=.5 \mathrm{e} 0 \mathrm{e} 924 \mathrm{~b} 61 \mathrm{ff} ;$

52. Thomée S. ICT use and mental health in young adults. Effects of computer and mobile phone use on stress, sleep disturbances, and symptoms of depression. Dissertation. Occupational and Environmental Medicine Department of Public Health and Community Medicine Institute of Medicine at Sahlgrenska Academy, University of Gothenburg 2012.

53. Thomée S, Härenstam A, Hagberg M. Mobile phone use and stress, sleep disturbances, and symptoms of depression among young adults - a prospective cohort study. BMC Public Health 2011; $11: 66$.

54. Twenge JM. iGen. Why today's super-connected kids are growing up less rebellious, more tolerant, less happy - and completely unprepared for aduldhood. New York: Atria Books 2017. 
55. Twenge JM. Have smartphones destroyed a generation? The Atlantic, September 2017 https://www. theatlantic.com/magazine/archive/2017/09/hasthe-smartphone-destroyed-a-generation/534198

56. Twenge JM, Joiner TE, Rogers ML, Martin GN. Increases in depressive Symptoms, suicide-related outcomes, and suicide rates among U.S. adolescents after 2010 and links to increased new media screen time. Clinical Psychological Science 2018; 6: 3-17.

57. Vom Orde H, Durner A. Grunddaten Jugend und Medien 2017. Aktuelle Ergebnisse zur Mediennutzung von Jugendlichen in Deutschland. Inter- nationales Zentralinstitut für das Jugend- und Bildungsfernsehen (IZI), München http://www.bronline.de/jugend/izi/deutsch/Grundddaten_Jugend_Medien.pdf

58. Wang Y, Xue H, Huang Y, Huang L, Zhang D. A systematic review of application and effectiveness of mHealth Interventions for Obesity and Diabetes Treatment and Self-Management. Adv Nutr 2017; 8: 449-462.

59. Witt K, Spittal MJ, Carter G, Pirkis J, Hetrick S, Currier D, Robinson J, Milner A. Effectiveness of online and mobile telephone applications (,apps') for the self-management of suicidal ideation and self-harm: a systematic review and meta-analysis. BMC Psychiatry 2017; 17: 297.

60. Wolniewicz CA, Tiamiyu MF, Weeks JW, Elhai JD. Problematic smartphone use and relations with negative affect, fear of missing out, and fear of negative and positive evaluation. Psychiatry Res pii: 2017; S0165-1781(17)30901-0.

61. Zhao J, Freeman B, Li M (2016) Can mobile phone apps influence peoples health behavior change? An Evidence Review. J Med Internet Res 2016; 18(11): e287.

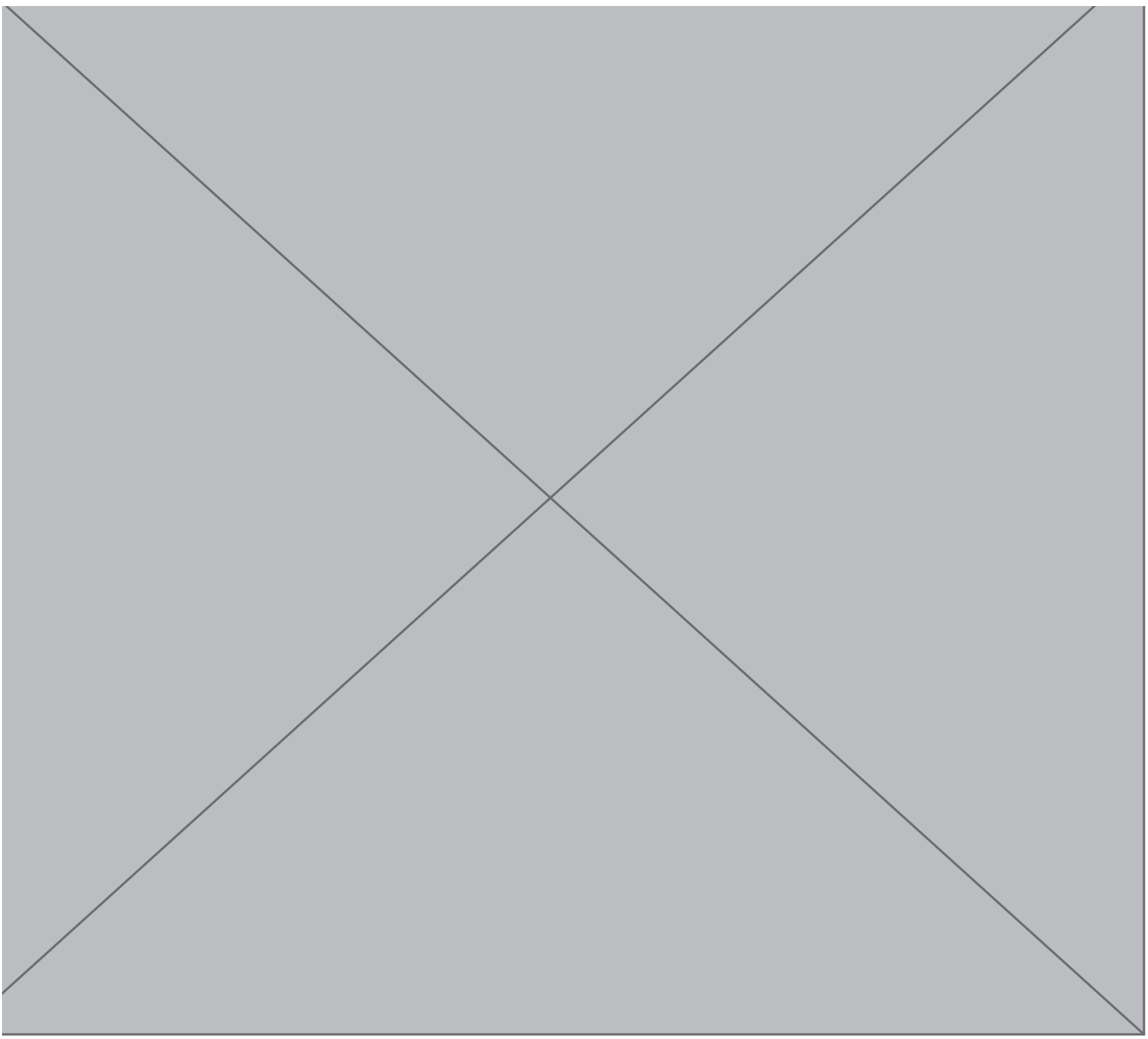

